\title{
Does the EQ-5D usual activities dimension measure what it intends to measure? The relative importance of work, study, housework, family or leisure activities
}

\author{
Thor Gamst-Klaussen ${ }^{1}$ (D) . Admassu N. Lamu ${ }^{2}$ D
}

Accepted: 7 April 2020 / Published online: 23 April 2020

(c) The Author(s) 2020

\begin{abstract}
Background The EQ-5D is the most widely used generic preference-based health-related quality of life measure. It comprises five dimensions: mobility, self-care, usual activities, pain/discomfort and anxiety/depression. The usual activities dimension asks respondents to evaluate the severity of problems in their usual activities, such as work, study, housework, family or leisure activities. The primary aim of this study is to investigate whether the EQ-5D (five-level) usual activities dimension captures those activities that it intends to capture. We further assess the relative importance of each of these activities for the usual activities dimension.

Methods Data include 7933 respondents from six countries: Australia, Canada, Germany, Norway, the UK, and the US. Logistic regression and ordinary least square regression models investigate the relationship between the usual activities dimension and its main predictors (work/study, housework, family, and leisure activities). A Shapley value decomposition method was applied to measure the relative importance of each predictor.

Results Work/study, housework, family, and leisure activities were all significant $(p<0.001)$ determinants of usual activities dimension. The respective marginal contribution (in \%) of housework, leisure, work/study and family to UA dimension (as a share of goodness-of-fit) is 28.0, 26.2, 20.8 and 14.7 in the logistic regression model. This finding is consistent when linear regression is used as an alternative model.

Conclusions The usual activities dimension in EQ-5D reflects the specific activities described to respondents. Therefore, the usual activities dimension measures what it really intends to measure.
\end{abstract}

Keywords EQ-5D $\cdot$ Usual activities $\cdot \mathrm{HRQoL} \cdot$ Shapley value $\cdot$ Relative importance

\section{Introduction}

Nowadays, the Euroqol 5-dimensional questionnaire, usually termed as EQ-5D, is the most widely used generic preference-based health-related quality of life measure [1-3]. Although EQ-5D has been developed to produce

Electronic supplementary material The online version of this article (https://doi.org/10.1007/s11136-020-02501-w) contains supplementary material, which is available to authorized users.

Thor Gamst-Klaussen

thor.klaussen@uit.no

1 Department of Psychology, UiT The Arctic University of Norway, 9037 Troms $\varnothing$, Norway

2 Department of Global Public Health and Primary Care, University of Bergen, 5020 Bergen, Norway a preference-based index for economic evaluations, it has been widely used across different settings, such as individual health status for use in clinical settings, observational studies, population health surveys [3]. The EQ-5D descriptive system initially comprised of six dimensions that included two dimensions asking respondents to report problems with: (i) 'Main activity' i.e., able to perform main activity (e.g., work, study, housework); and (ii) 'Social relationships' i.e., able to pursue family and leisure activities, as well as the other four dimensions of the current version of EQ-5D (mobility, self-care, pain/discomfort, and anxiety/depression) [4]. However, the initial experimentation with this six-dimensional version resulted in the merging of 'Social relationships' with 'Main activity' dimension that became the current 'UA' dimension, which leads to the present five-dimensional questionnaire. This change was accompanied by an explicit description of 
the UA dimension as work, study, and housework alongside family and leisure activities [4]. There was a broad agreement amongst the EuroQol group that the UA dimension would cover the described daily activities, where 'usual' was perceived as referring to some frequency or regularity of performance of the activities [5].

Nevertheless, practitioners have raised concerns about the UA dimension due to either respondents' perceived ambiguity when interpreting the UA dimension or respondents' counter-intuitive responses [5]. Selai [5] summarized possible sources of ambiguity, including semantics (usual/unusual may refer to regularity/frequency or to normal versus abnormal); vague quantifiers (relative frequency conveys different meaning depending on race, education, and age); and the double or multi-barrelled nature of the question (e.g., how to answer if you cannot work but do not have any problems doing leisure activities like reading or playing chess). Furthermore, in adaption to long-lasting chronic illness, one's usual activities that were initially affected by the disease become less regular and replaced by new daily or frequent activities through activity adjustment [6]. This might make the UA dimension especially prone to adaption that would potentially affect the responses of individuals with chronic conditions.

In general, the UA dimension is thought to measure both activities and social participation [7, 8]. However, there is mixed evidence from the literature. For example, research among stroke patients suggested that the UA dimension was measuring social functioning [5,9], while a study among patients with diabetes indicated that the UA dimension measure limitation with work but not social activities [10]. Lin et al. [11] also showed that UA dimension correlated strongly with subscales on the Patient-Reported Outcome Measurement Information System (PROMIS), such as physical function, fatigue, and satisfaction with social roles. It has also been suggested that interpersonal relationships might be important as a bolt-on dimension [12, 13].

Moreover, evidence showed that the UA dimension is associated with both physical and mental aspects of health, but more strongly with the former. For instance, using data comprising seven disease groups and a nondiagnosed healthy group, Gamst-Klaussen et al. revealed stronger correlations between UA dimension and the other EQ-5D dimensions that tap physical health (i.e., mobility, self-care and pain/discomfort) than with anxiety/depression [14]. A similar result was found in a study among individuals with COPD [11]. A study that described the most commonly reported EQ-5D health states using SF-36 in the general Swedish population showed that moderate problems on EQ-5D UA dimension most strongly affected the SF-36 role limitations scales due to physical health problems [15]. A comparison of SF-12 and EQ-5D administered in the US general population also demonstrated that the UA dimension had a stronger relationship with the SF-12 physical health component than the SF-12 mental health component [16].

The UA dimension generally reflects a wider concept that may include several activities. The important question is whether the UA dimension captures activities that are described in the EQ-5D questionnaire. To the best of our knowledge, no study has investigated this research question. Thus, the primary aims of the current study are twofold: (i) to investigate whether the described activities (e.g. work, study, housework, family or leisure activities) actually determine the EQ-5D UA dimension; and (ii) to assess the relative importance of each of these activities for the UA dimension. We also check the consistency of our findings using samples from seven disease groups and the healthy group.

\section{Method}

\section{Data}

Data were obtained from the multi-instrument comparison (MIC) study, which is based on a 2011/2012 online survey from six countries (Australia, Canada, Germany, Norway, UK, US) and administered by a global panel company, CINT Pty Ltd. The data include a sample of 7933 respondents comprising a 'healthy group' $(N=1760)$ and seven major disease groups $(N=6173)$. Respondents were initially asked to indicate if they had a chronic disease and to rate their overall health on a visual analogue scale (VAS). Quotas on age, gender, and education were used to obtain a demographically representative sample of a healthy group, defined by the absence of chronic disease and a VAS score of at least 70 on overall health. Quotas were also applied to obtain a target number of respondents in each disease group (arthritis, asthma, cancer, depression, diabetes, hearing loss, heart diseases). See Table 1 for the description of data.

The MIC survey includes six generic preference-based measures and a generic health status measure, as well as several disease-specific and well-being measures. To avoid priming the respondents' feelings with questions about their health, which could potentially lead to biased responses, the subjective well-being questions were first administered. Next, EQ-5D and other generic measures were administered in a randomized order to avoid order effects. Finally, the respondents in each disease-group completed a disease-specific measure. Responses were subject to several stringent edit procedures based upon a comparison of duplicated or similar questions as well as a minimum completion time to ensure the quality of data. The detailed edit procedures and its administrations are available elsewhere [17]. 
Table 1 Number of respondents by country and disease group

\begin{tabular}{lcrrrrrrr}
\hline Diseases & Australia & UK & USA & Canada & Norway & Germany & Total & $\%$ \\
\hline Asthma & 141 & 150 & 150 & 138 & 129 & 147 & 855 & 10.7 \\
Cancer & 154 & 137 & 148 & 138 & 80 & 115 & 772 & 10.0 \\
Depression & 146 & 158 & 168 & 145 & 140 & 160 & 917 & 12.0 \\
Diabetes & 168 & 161 & 168 & 144 & 143 & 140 & 924 & 12.0 \\
Hearing loss & 155 & 126 & 156 & 144 & 113 & 136 & 830 & 10.5 \\
Arthritis & 163 & 159 & 179 & 139 & 130 & 159 & 929 & 12.0 \\
Heart disease & 149 & 167 & 170 & 154 & 151 & 152 & 943 & 12.0 \\
Healthy group & 265 & 298 & 321 & 328 & 288 & 260 & 1760 & 22.0 \\
Total & 1341 & 1356 & 1460 & 1330 & 1174 & 1269 & 7933 & 100.0 \\
\hline
\end{tabular}

\section{Measures of variables}

\section{Outcome variable}

The EQ-5D has five dimensions: mobility, self-care, usual activities, pain/discomfort, and anxiety/depression. Each dimension has three severity levels in the original version and five severity levels in the revised version. Here, we considered the latter version, EQ-5D five-levels. The UA dimension of EQ-5D is the outcome or dependent variable and has five response levels: no problems, slight problems, moderate problems, severe problems, extreme problems. A closer examination reveals that all except the first level have one common feature: having problems with usual activities (though with different degrees of severity). Thus, the UA dimension can logically be dichotomized into full health and impaired health. That is, level-1_- "I have no problems doing my usual activities"-represents full health, while the rest (level-2 to level-5) represent impaired health.

\section{Predictor variables}

Relevant items were identified from Medical Outcomes Study 36-item Short-Form (SF-36) questionnaire and the Assessment of Quality of Life (AQoL) questionnaire that would likely measure the main predictors: work/study, housework, family, and leisure activities.

Work/study activities Seven items from SF-36 were selected, which would likely measure problems related to work/study activities due to one's health. Four items were related to physical health, and the remaining three items ask about emotional problems. The question related to physical health is described as: During the past 4 weeks, have you had any of the following problems with your work or other regular daily activities as a result of your PHYSICAL health?

(i) Cut down the amount of time you spent on work or other activities; (ii) Accomplished less than you would like

(iii) Were limited in the kind of work or other activities;

(iv) had difficulty performing work or other activities (for example, it took extra effort).

The emotional question is stated as: During the past 4 weeks, have you had any of the following problems with your work or other regular daily activities as a result of your EMOTIONAL problems (such as feeling depressed or anxious)?

(i) Cut down the amount of time you spent on work or other activities;

(ii) Accomplished less than you would like;

(iii) Did not do work or other activities as carefully as usual.

Responses for each of the seven items from physical and emotional health is given on a five-point Likert scale (reverse coded): None of the time; A little of the time; Some of the time; Most of the time; All of the time. The total summary score of the seven items was used as a measure of work/study activities and treated as a continuous variable with higher values indicating greater problems in performing work/study activities. To check the consistency of our results, one item from EORTC Quality of Life Questionnaire (QLQ C-30) with a similar measure of work/study activity was applied using a cancer sample $(N=772)$. It is described as: were you limited doing either your work or other daily activities? (Not at all; A little; Quite a bit; Very much).

Housework activities One item from AQoL with five response levels was selected to measure housework activities, and defined as: How much help do you need with jobs around the house (e.g., preparing food, cleaning the house or gardening)? The responses include: I can do all these tasks very quickly and efficiently without any help; I can do these tasks relatively easily without help; I can do these tasks only very slowly without help; I cannot do most of 
these tasks unless I have help; I can do none of these tasks by myself.

Because only a few responses were observed on the last scale $(0.5 \%)$, the last two levels were combined. Thus, housework is treated as a categorical variable with four levels.

Family activities One item from AQoL was selected to measure family activities. The item has four response levels, and described as follows Thinking about your health and your relationship with your family; my role in the family is unaffected by my health; there are some parts of my family role I cannot carry out; there are many parts of my family role I cannot carry out; I cannot carry out any part of my family role. The two most severe levels were merged due to few respondents on the last level (1.3\%).

Leisure activities Two items from SF-36 (each with three response levels) were selected as a measure of leisure activities. That is, respondents are asked to consider if their health limits them in the following activities, and if so how much.

(i) Vigorous activities, such as running, lifting heavy objects, participating in strenuous sports (Yes, limited a lot; Yes, limited a little; Not limited at all).

(ii) Moderate activities, such as moving a table, pushing a vacuum cleaner, bowling, or playing golf (Yes, limited a lot; Yes, limited a little; Not limited at all).

The total score was calculated by summing the reversecoded responses across the two items. Eventually, this variable is grouped into five categories ranging from 1 (not limited at all) to 5 (limited a lot) in the full sample. Because only a few responses were observed on the first level, the first two levels were combined in the sample of the healthy group, asthma, and hearing problems.

The descriptions of these two items go beyond leisure activities because they illustrate physical and housework activities as well. Thus, to check the consistency of our results, a similar analysis was conducted using a sample of cancer patients $(N=772)$ who reported their HRQoL using QLQ C-30 that includes one item with a better measure of leisure activity. The item asks specifically about hobbies and other leisure activities and has four response levels. It is described as: were you limited pursuing your hobbies or other leisure activities? (Not at all; A little; Quite a bit; Very much).

Control variables In addition to the described main predictors, we considered the effect of socio-demographic characteristics. Age is included as a continuous variable to control any variation in UA due to age differences. Gender $(0=$ male; $1=$ female $)$ is used to control sex differences.
Marital status may also be an important determinant of UA $(0=$ no partner/spouse, $1=$ partner/spouse $)$. Education level is accounted for by dummies $(0=$ high school; $1=$ diploma or certificate; $2=$ university). Employment status is dichotomized (unemployed vs. all others) to reflect the evidence that being unemployed has a particularly adverse effect on usual activities dimension. Furthermore, disease variables are included since they may signal the effect of health variations on UA and country variables to capture country-specific heterogeneity. Description of variables is summarized in Table 2.

Table 2 Description of variables

\begin{tabular}{|c|c|c|}
\hline Variable & Mean $/ n$ & $\mathrm{SD} / \%$ \\
\hline Age (years) & 51.46 & 15.41 \\
\hline \multicolumn{3}{|l|}{ Gender, $n(\%)$} \\
\hline Female & 4140 & 52.19 \\
\hline Male & 3793 & 47.81 \\
\hline \multicolumn{3}{|l|}{ Marital status, $n(\%)$} \\
\hline Live with partner/spouse & 5085 & 64.1 \\
\hline Do not live with partner/spouse & 2848 & 35.9 \\
\hline \multicolumn{3}{|l|}{ Education level, $n(\%)$} \\
\hline High school & 2482 & 31.29 \\
\hline Diploma/certificate & 3208 & 40.44 \\
\hline University & 2243 & 28.27 \\
\hline \multicolumn{3}{|l|}{ Employment status, $n(\%)$} \\
\hline Unemployed & 607 & 7.65 \\
\hline Employed/other & 7323 & 92.35 \\
\hline \multicolumn{3}{|l|}{ Usual activities, $n(\%)$} \\
\hline Have no problems & 5163 & 65.08 \\
\hline Have problems & 2770 & 34.92 \\
\hline Work/study & 13.40 & 7.60 \\
\hline \multicolumn{3}{|l|}{ Housework, $n(\%)$} \\
\hline Can do all tasks very quickly & 3912 & 49.31 \\
\hline Can do relatively easily & 2475 & 31.2 \\
\hline Can do very slowly & 1079 & 13.6 \\
\hline Cannot do most unless help/none & 467 & 5.89 \\
\hline \multicolumn{3}{|l|}{ Family, $n(\%)$} \\
\hline My family role is unaffected & 5288 & 66.66 \\
\hline I cannot carry out some parts & 1957 & 24.67 \\
\hline I cannot carry out many parts/any parts & 688 & 8.67 \\
\hline \multicolumn{3}{|l|}{ Leisure, $n(\%)$} \\
\hline Not limited & 2095 & 26.41 \\
\hline Slightly limited & 2148 & 27.08 \\
\hline Somewhat limited & 1279 & 16.12 \\
\hline Moderately limited & 1516 & 19.11 \\
\hline Limited a lot & 894 & 11.27 \\
\hline
\end{tabular}




\section{Statistical analyses}

Respondents' characteristics were described as mean (standard deviation, SD) unless otherwise indicated. The Spearman rank-order correlation analysis was used to test the association between UA dimension and the main predictors. Spearman's correlation coefficient $(\rho)$ measures the strength and direction of the monotonic association between two variables. It is a nonparametric rank statistic often used to evaluate relationships involving ordinal variables without making any assumptions about the frequency distribution of the variables [18].

Logistic regression model (LRM), which is the most commonly used for a binary outcome, was applied to investigate the effect of main predictors (work/study, housework, family, and leisure activities) on UA dimension of EQ-5D. Standard ordinary least square (OLS) regression results were also presented to facilitate comparison with the LRM, which enables us to test the stability of our results. In the OLS, the UA dimension with five severity levels was used as a continuous variable. All explanatory variables were tested for multi-collinearity and were found to be satisfactory as their maximum Variance Inflation Factors (VIFs) were below 3.0, which is much less than the generally accepted maximum threshold value of 10 [19].

In both models, we first tested the significance of the main predictors in explaining the UA dimension. Then we investigated the relative contribution of each of the main predictors for UA dimension using the variance decomposition approach. The variance decomposition method, referred to as the Shapley value (SV) regression, was employed to detect the relative importance of each predictor (work/study, housework, family, and leisure activities) for UA dimension. In health research with inherently imprecise measures of complex concepts such as quality of life or self-reported health, a correlation among predictors is often the norm [20]. Therefore, the SV regression [21] is a reliable and stable method for the estimation of predictor importance even in the presence of high multi-collinearity. The SV measures the marginal contribution to the explained variance $\left(R^{2}\right)$ from adding a given independent variable to the model, weighted by the number of permutations represented by this sub-model [22]. A detailed description of SV is found elsewhere [20, 22].

Model estimates from a logistic regression are based on maximum likelihood estimates retrieved through an iterative process. Unlike the OLS $R^{2}$, several pseudo $R^{2}$ have been developed to evaluate the goodness-of-fit of logistic models. Because the interpretation of pseudo $R^{2}$ is not identical to the $R^{2}$ in OLS regression (the proportion of variance explained by the predictors), we suggest caution in interpreting it. Nevertheless, in this study, we used McFadden's pseudo $R^{2}$ [23], which is the most commonly used and more straightforward in the sense that it reflects both the criterion being minimized in logistic regression estimation and the variance-accounted for by the LRM [24]. Although the Pseudo- $R^{2}$ from LRM is not directly comparable with the OLS $R^{2}$, the SV approach can decompose the total explained variation from both LRM and OLS.

\section{Results}

Summary statistics of the variables were presented in Tables 1 and 2. Almost $65 \%$ of individuals reported no problems in doing their UA activities, while those with no problems in family, housework, leisure, and work/study activities was $66.7,49.3,30.7$, and $26.4 \%$, respectively. The mean age of respondents was $51.46(\mathrm{SD}=15.41)$ years, and ranged 18-93 years. Data were fairly gender-balanced (52\% female). Most respondents had a diploma or university degree $(68 \%)$, the majority lived with a partner or spouse (64\%), and the distributions of chronic diseases were fairly balanced in the sample.

The Spearman correlation coefficient between the UA dimension and the main predictors were quite high: with work/study (rho, $\rho=0.63$ ), housework $(\rho=0.66$ ), family $(\rho=0.57)$ and leisure activities $(\rho=0.63)$. The Spearman's correlation coefficient tends to yield a better correlation estimate than a Pearson product-moment correlation applied to ordinal variables, especially when the distribution of the ordinal variables is skewed.

The regression results were presented in Table 3. All main predictors, including work/study, housework, family, and leisure activities were significant $(p<0.001)$ determinants of UA dimension. For instance, results from the LRM revealed that the odds of having problems in UA was $12\left(=e^{2.5}\right)$ times higher for those who are unable to do the housework tasks than those who are able to do the housework tasks very quickly. Similarly, OLS results showed that being unable to do most or none of the housework tasks without help leads to 0.85 higher problems in doing UA as compared to those who can do housework tasks very quickly. In the OLS, being 'slightly limited' to do leisure activities reduced UA by 0.6 -percentage point as compared to 'not limited at all' but was not statistically significant; however, the LRM significantly picked up the difference. Among control variables, having a chronic condition significantly determined UA. Both LRM and OLS models generally produced similar results in the sense that in both models the regression results showed the same directions. Both models also produced similar ranks with regard to the importance of activities that describe the UA dimension (as detailed in the next paragraph). Results from the cancer sample with a more precise definition of leisure variable demonstrated 
Table 3 Regression results for usual activities dimension

\begin{tabular}{|c|c|c|c|c|c|c|c|c|}
\hline \multirow[t]{3}{*}{ Variables } & \multicolumn{4}{|l|}{ Model 1} & \multicolumn{4}{|l|}{ Model 2} \\
\hline & \multirow[t]{2}{*}{$\beta$} & \multirow[t]{2}{*}{ S.E. } & \multicolumn{2}{|l|}{$95 \% \mathrm{CI}$} & \multirow[t]{2}{*}{$\beta$} & \multirow[t]{2}{*}{ S.E. } & \multicolumn{2}{|l|}{$95 \% \mathrm{CI}$} \\
\hline & & & Lower & Upper & & & Lower & Upper \\
\hline Work/study & $0.093 * * *$ & 0.006 & 0.08 & 0.105 & $0.027 * * *$ & 0.001 & 0.024 & 0.029 \\
\hline \multicolumn{9}{|l|}{ Housework } \\
\hline Can do relatively easy & $1.011 * * *$ & 0.085 & 0.845 & 1.178 & $0.124 * * *$ & 0.015 & 0.095 & 0.152 \\
\hline Can do very slowly & $2.186^{* * *}$ & 0.132 & 1.927 & 2.445 & $0.568 * * *$ & 0.031 & 0.507 & 0.630 \\
\hline Cannot do most/none & $2.475 * * *$ & 0.246 & 1.993 & 2.957 & $0.853 * * *$ & 0.051 & 0.753 & 0.954 \\
\hline \multicolumn{9}{|l|}{ Family } \\
\hline Some parts affected & $0.776^{* * *}$ & 0.083 & 0.612 & 0.939 & $0.133 * * *$ & 0.019 & 0.095 & 0.170 \\
\hline Many/cannot cary any & $0.934 * * *$ & 0.165 & 0.612 & 1.257 & $0.469 * * *$ & 0.04 & 0.391 & 0.548 \\
\hline \multicolumn{9}{|l|}{ Leisure } \\
\hline Slightly limited & $0.639 * * *$ & 0.127 & 0.392 & 0.887 & 0.006 & 0.011 & -0.016 & 0.028 \\
\hline Somewhat limited & $1.314 * * *$ & 0.133 & 1.053 & 1.575 & $0.093 * * *$ & 0.019 & 0.056 & 0.130 \\
\hline Moderately limited & $2.014 * * *$ & 0.135 & 1.748 & 2.279 & $0.273 * * *$ & 0.024 & 0.227 & 0.319 \\
\hline Limited a lot & $2.104 * * *$ & 0.185 & 1.740 & 2.467 & $0.588 * * *$ & 0.041 & 0.507 & 0.668 \\
\hline Age (in years) & -0.0005 & 0.003 & -0.006 & 0.005 & -0.0001 & 0.0004 & -0.001 & 0.001 \\
\hline \multicolumn{9}{|l|}{ Gender } \\
\hline Female & -0.028 & 0.076 & -0.177 & 0.121 & -0.013 & 0.012 & -0.036 & 0.011 \\
\hline \multicolumn{9}{|l|}{ Marital } \\
\hline Live with spouse/partner & -0.084 & 0.075 & -0.231 & 0.064 & -0.012 & 0.012 & -0.036 & 0.013 \\
\hline \multicolumn{9}{|l|}{ Education } \\
\hline Diploma/certificate & $-0.140 *$ & 0.085 & -0.306 & 0.026 & $-0.026^{*}$ & 0.014 & -0.054 & 0.002 \\
\hline University & $-0.226^{* *}$ & 0.095 & -0.412 & -0.039 & $-0.037 * *$ & 0.015 & -0.066 & -0.008 \\
\hline \multicolumn{9}{|l|}{ Employment status } \\
\hline Unemployed & 0.097 & 0.139 & -0.175 & 0.368 & 0.017 & 0.026 & -0.033 & 0.068 \\
\hline \multicolumn{9}{|l|}{ Disease } \\
\hline Arthritis & $1.284 * * *$ & 0.146 & 0.998 & 1.571 & $0.149 * * *$ & 0.022 & 0.106 & 0.192 \\
\hline Asthma & $0.628 * * *$ & 0.152 & 0.330 & 0.925 & $0.051 * * *$ & 0.018 & 0.015 & 0.087 \\
\hline Cancer & $0.816^{* * *}$ & 0.152 & 0.518 & 1.114 & $0.107 * * *$ & 0.022 & 0.063 & 0.151 \\
\hline Depression & $0.925 * * *$ & 0.154 & 0.623 & 1.226 & $0.088 * * *$ & 0.025 & 0.039 & 0.136 \\
\hline Diabetes & $0.543 * * *$ & 0.148 & 0.253 & 0.833 & $0.048 * *$ & 0.019 & 0.010 & 0.085 \\
\hline Hearing & $0.500 * * *$ & 0.162 & 0.182 & 0.819 & $0.032 *$ & 0.018 & -0.003 & 0.067 \\
\hline Heart & $0.738 * * *$ & 0.147 & 0.450 & 1.026 & $0.079 * * *$ & 0.02 & 0.040 & 0.119 \\
\hline \multicolumn{9}{|l|}{ Country } \\
\hline Australia & $-0.229^{*}$ & 0.127 & -0.477 & 0.020 & $-0.102 * * *$ & 0.02 & -0.142 & -0.062 \\
\hline Canada & -0.122 & 0.129 & -0.376 & 0.132 & $-0.037^{*}$ & 0.02 & -0.076 & 0.002 \\
\hline Germany & $0.300 * *$ & 0.129 & 0.048 & 0.553 & 0.033 & 0.021 & -0.008 & 0.074 \\
\hline Norway & 0.178 & 0.132 & -0.081 & 0.437 & 0.003 & 0.02 & -0.036 & 0.042 \\
\hline USA & -0.139 & 0.126 & -0.387 & 0.108 & $-0.053 * * *$ & 0.02 & -0.092 & -0.014 \\
\hline Constant & $-10.700 * * *$ & 0.583 & -11.843 & -9.557 & $-0.141 * * *$ & 0.077 & -0.2923 & 0.011075 \\
\hline
\end{tabular}

Logistic regression is used in Model 1, and linear regression in Model 2

$\beta$ estimated coefficients, S.E. standard error, $C I$ confidence interval

$* * * p<0.01, * * p<0.05, * p<0.1$

similar results (Online Appendix Table A1), and so does the proxy variable for work/study (Online Appendix Table A2).

Figure 1 and Table 4 summarize the relative importance of each predictor for UA dimension of EQ-5D. The overall goodness-of-fit was over 49 and 63\% in LRM and OLS, respectively. Housework, leisure, work/study, and family activities significantly contributed to the total explained variation in the UA dimension, in that order. For instance, 
Fig. 1 Relative importance of predictors in the full sample $(N=7933)$. Note: $S D C$ Sociodemographic characteristics (age, gender, marital, education and unemployment)

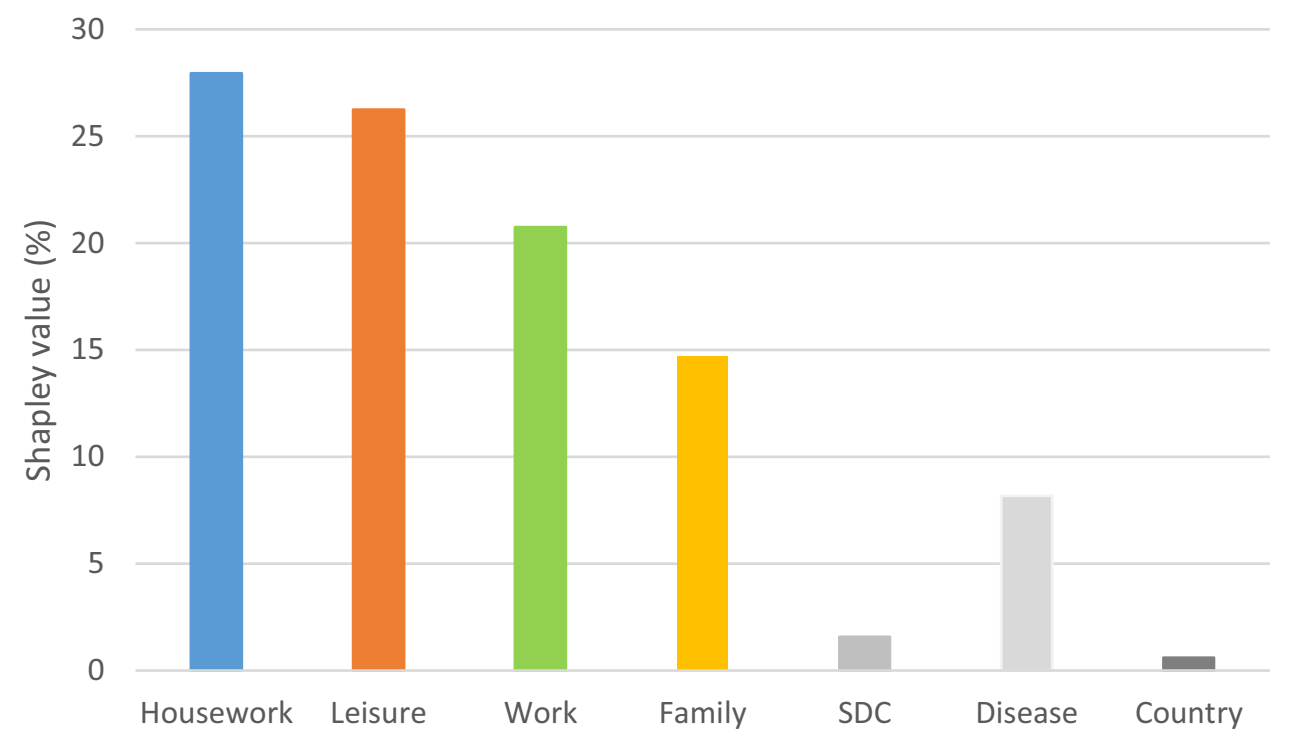

Table 4 Relative importance of predictors for EQ-5D usual activities dimension

\begin{tabular}{|c|c|c|c|c|}
\hline \multirow[t]{3}{*}{ Predictors } & \multicolumn{4}{|c|}{ Full sample $(n=7933)$} \\
\hline & \multicolumn{2}{|c|}{ Model 1} & \multicolumn{2}{|c|}{ Model 2} \\
\hline & Est & Percent & Est & Percent \\
\hline Work/study & 0.102 & 20.75 & 0.145 & 22.85 \\
\hline Housework & 0.137 & 27.95 & 0.182 & 28.74 \\
\hline Family & 0.072 & 14.71 & 0.113 & 17.89 \\
\hline Leisure & 0.129 & 26.24 & 0.153 & 24.11 \\
\hline SDC & 0.008 & 1.58 & 0.007 & 1.07 \\
\hline Disease & 0.040 & 8.18 & 0.030 & 4.73 \\
\hline Country & 0.003 & 0.60 & 0.004 & 0.62 \\
\hline Total & 0.491 & 100 & 0.633 & 100 \\
\hline
\end{tabular}

Model 1 applied logistic regression, and Model 2 linear regression

$S D C$ socio-demographic characteristics (age, gender, marital, education abd unemployment), Est. estimated shapley value

the respective marginal contribution of housework, leisure, work/study, and family activities to UA dimension (as a share of goodness-of-fit) was 28.0, 26.2, 20.8, and $14.7 \%$ in the LRM. Similar results observed in the OLS regression model: housework contributed the highest (28.7\%) followed by leisure $(24.1 \%)$. Family activities contributed the least $(17.9 \%)$.

Analysis using a sample of cancer patients $(N=772)$ with a more precise measure of leisure activities produced similar results, except that work/study became the secondmost important for UA dimension instead of leisure (Online Appendix Table A3, Scenario 1). In the LRM, the relative contributions of housework, work/study, leisure, and family activities to UA dimension of the EQ-5D were 37.8, $23.4,19.9$, and $15.9 \%$, respectively. A similar pattern was observed from the OLS regression model. When we consider an alternative measure of work/study variable from the cancer sample, the LRM produced identical importance rankings; i.e., housework is the most important variable followed by leisure similar to the model with full sample. Family variable ranked last. However, leisure and work/study switched in the OLS model. For detail, see Online Appendix Table A3.

Figure 2 and Online Appendix Table A4 summarized the relative importance of the main predictors of UA dimension across the healthy group and disease groups. The relative importance rankings were quite similar across different groups. Housework was the most important variable except in the arthritis group, where leisure contributed most. Family activities consistently ranked least except in the healthy group where work/study and family activities switched importance rankings. In addition, the depression group differs in two ways: first, control variables were most strongly associated with the UA dimension; and second, it had the lowest goodness-of-fit (i.e., $R^{2}=38 \%$ ). It is also worth mentioning that the marginal contribution of work/study exceeds that of leisure in the depression group alone.

\section{Discussion}

This study investigated whether the EQ-5D usual activities dimension reflects measures of variables related to work/ study, housework, family, and leisure activities as described in the questionnaire. The findings suggest that respondents consider these wide ranges of activities when reporting on how their health affects their UA, and hence the UA dimension measures what it intends to measure. Inquiry into 
Fig. 2 Relative importance of predictors in disease groups and healthy group. Note: $S D C$ Socio-demographic characteristics (age, gender, marital, education and unemployment)

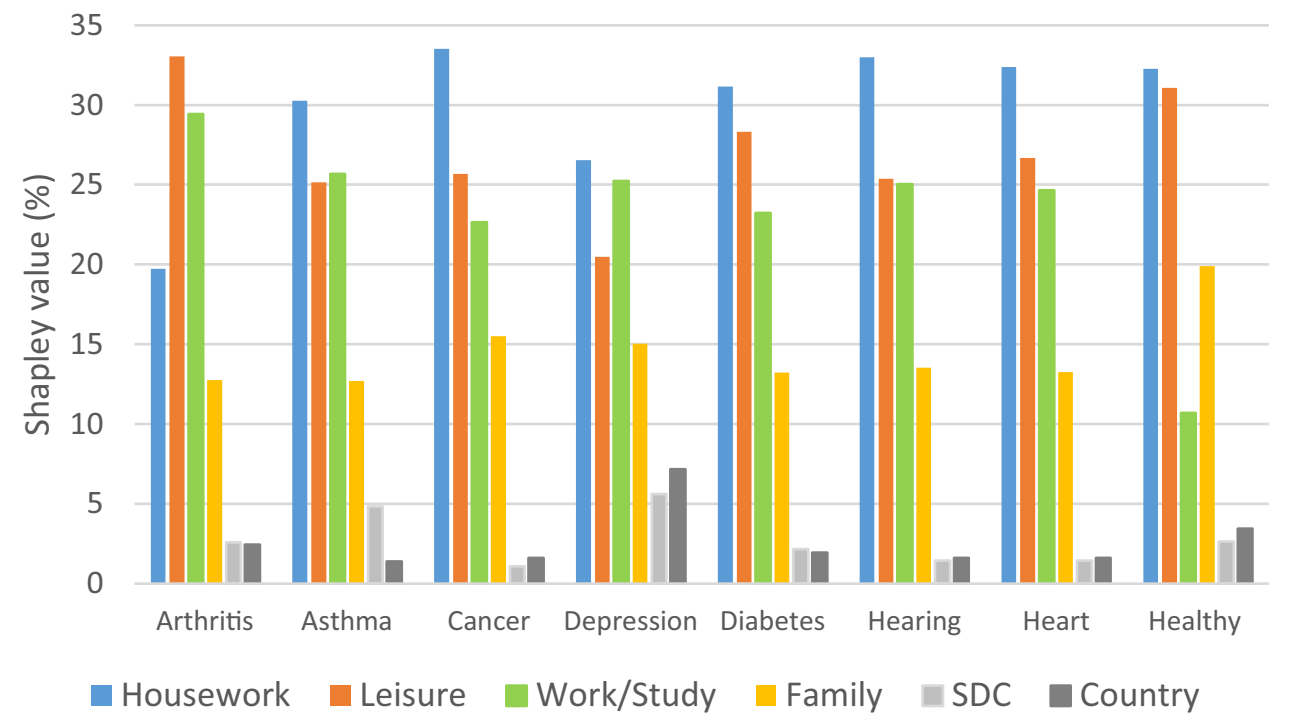

relative importance revealed that housework explains most of the overall variance, followed by leisure, while family activities ranked last.

This sequence of importance ranking was consistent across the majority of disease groups as well as the healthy group, with housework at the top. This finding suggests that problems with housework are more salient and possibly more difficult to adapt too. That is, change in some activities might be less obvious to the individual (e.g., leisure activities change from playing squash to playing chess), but it is difficult to adapt to an inability to do basic housework activities like preparing food or cleaning the house. Another reason for the dominant role of housework might be that the term "usual" is commonly understood as "daily" by respondents [25], which could make other non-daily regular activities (e.g., problems playing golf once a week or meeting family once a month) less likely to be reported when considering one's usual activities.

Compared to family activities, housework activities contributed twice as much of the overall variation. The possible explanation for the lower contribution of family activities could be an overlap with housework and leisure activities. In the description of the EQ-5D UA dimension, there is no clear definition as what a 'family activity' means. Thus, respondents may attribute some of the activities related to family as housework or leisure activities. In fact, it is difficult to draw a line of demarcation between family activities and housework or leisure activities. For instance, cooking can be both housework and family activities as well as leisure activities. Moreover, people may understand family activities as social phenomena instead of usual activities that are more physical, such as housework, which would be in line with the original EQ-6D that described family and leisure activities under the social relationship dimension [4].
The leisure variable is the second-most important for UA dimension that even exceeds the contribution of work/study. The reason why the relative importance of leisure activities generally exceeds that of work/study is most probably that leisure activities extend from the very informal and casual to highly organized and long-lasting activities, while work/ study are specific and well defined for each individual.

In the healthy and disease groups, the relative importance ranking of the main predictors of UA dimension was consistent except in the arthritis group. In the arthritis group, leisure activity was most important, followed by work/study, housework, and family. This result can be explained by the nature of the disease and the way the leisure variable was described. That is, the leisure activities variable focuses on the ability to do vigorous activities such as participating in strenuous sports or lifting heavy objects, as well as moving a table or pushing a vacuum challenging to individuals with arthritis. In contrast, in the depression group, leisure, and work/study activities switched importance rankings. This result is likely because of the nature of the leisure variable; leisure is tapping health that is more physical. However, the items used to measure work/study include items that explicitly emphasize emotional problems (i.e., feeling depressed or anxious), which could increase the contribution of work/study in the depression group. For instance, a separate regression analysis (not reported here) with work/study activities as two separate variables (emotional health and physical health) revealed that only the latter significantly affect the UA dimension, suggesting that respondents mainly focus on their physical health when reporting problems on the UA. Furthermore, although depression affects motivation to do usual activities, both housework and leisure (as described in this study) are mostly measuring a physical cleaner, which are activities that might be especially 
concept. Lastly, for the healthy sample, housework and leisure activities continued to contribute the lion shares to the UA dimension, and work/study activities interestingly ranked last. However, this is not surprising, considering healthy people are usually less concerned about their work/study compared to people with chronic conditions.

It seems evident that the term usual is understood as activities that are performed with some regularity [25]. Thus, one area of ambiguity is the notion of how 'regular' is 'usual'; i.e., the ambiguity in the interpretation of the term 'usual' lies in whether 'usual' mean 'daily' or 'regular' (e.g., visiting families every week-end). Another area of ambiguity is whether 'usual' covers 'role' or other activities, and whether the individual is fulfilled or not, particularly in reference to activities preceeding illness [25]. Furthermore, Rand-Henriksen et al. compared hypothetical and experience based EQ-5D valuations and argued that usual activities could be the least tangible dimension for the general public [26]. The issue of valuation of EQ-5D health states is beyond the scope of the current study; however, the ambiguity in the interpretation of the term 'usual' in the UA dimension could complicate the valuation exercise and warrant future studies.

This study has a number of limitations. One limitation is the measure of leisure variable from the SF-36 questionnaire. As discussed, these items measure mainly vigorous activities (e.g., participating in strenuous sports), and has some overlap with housework. Nevertheless, our findings are consistent with a more precise measure of leisure (obtained from the QLQ-C30 instrument). Another limitation could be the lack of a clear distinction between the described activities, such as housework, leisure, and family activities, which highlights the challenge using such a multi-barreled question to measure usual activities. Further, not all variation in UA dimension is accounted for by the explanatory variables used in this study. This indicates that additional activities other than those described here are important for variation in UA. However, the four major activities alone explained over 44 and 59\% of the variation in LRM and OLS, respectively, which is quite large in this kind of social study. Lastly, respondents volunteered to participate in the MIC study, something which might lead to self-selection bias. Despite these limitations, this is the first study that has empirically attempted to test whether these activities, in fact, reflect the UA dimension of EQ-5D. Future studies should apply better measures of work/study, housework, family and leisure activities that can minimize conceptual overlap between these variables. Further studies would also be required to identify other important predictor(s) of the UA dimension, which can give insights into what respondents are considering when they report problems on this dimension.

In conclusion, the UA dimension fairly picks up the described activities, such as work/study, housework, family, and leisure activities. In explaining the variation in the
UA dimension, housework is relatively most important, followed by leisure, while family is the least important. In a nutshell, the UA dimension of EQ-5D captures what it intends to measure.

Acknowledgements Open Access funding provided by UiT The Arctic University of Norway. We would like to thank Professor Frode Svartdal and Professor Jan Abel Olsen for helpful discussions and useful contributions to the manuscript. The Australian National Health and Medical Research Council, Grant Number 1006334, funded data collection in five countries (Australia, Canada, Germany, UK, and the US) while UiT The Arctic University of Norway funded data collection for the Norwegian part.

Funding Data collection was funded by Grants from The Australian NHMRC (Grant Number 1006334) and the University of Tromsø.

\section{Compliance with ethical standards}

Conflict of interest The authors declare that they have no conflict of interest.

Ethical approval This article does not contain any studies with human participants performed by any of the authors.

Open Access This article is licensed under a Creative Commons Attribution 4.0 International License, which permits use, sharing, adaptation, distribution and reproduction in any medium or format, as long as you give appropriate credit to the original author(s) and the source, provide a link to the Creative Commons licence, and indicate if changes were made. The images or other third party material in this article are included in the article's Creative Commons licence, unless indicated otherwise in a credit line to the material. If material is not included in the article's Creative Commons licence and your intended use is not permitted by statutory regulation or exceeds the permitted use, you will need to obtain permission directly from the copyright holder. To view a copy of this licence, visit http://creativecommons.org/licenses/by/4.0/.

\section{References}

1. Wisløff, T., Hagen, G., Hamidi, V., Movik, E., Klemp, M., \& Olsen, J. A. (2014). Estimating QALY gains in applied studies: A review of cost-utility analyses published in 2010. Pharmacoeconomics, 32(4), 367-375.

2. Richardson, J. R., Mckie, J. R., \& Bariola, E. J. (2014). Multi attribute utility instruments and their use. In A. J. Culyer (Ed.), Encyclopedia of health economics (pp. 341-357). San Diego: Elsevier Science.

3. Devlin, N. J., \& Brooks, R. (2017). EQ-5D and the EuroQol group: Past, present and future. Applied Health Economics and Health Policy, 15(2), 127-137.

4. Gudex, C. (2005). The descriptive system of the EuroQol instrument. In P. Kind, R. Brooks, \& R. Rabin (Eds.), EQ-5D concepts and methods: A developmental history (pp. 19-27). Dordrecht: Springer.

5. Selai, C. (1998). The measurement precision of the EQ-5D domain "Usual activities", EuroQol Proceedings. Hannover: EuroQol.

6. Menzel, P., Dolan, P., Richardson, J., \& Olsen, J. A. (2002). The role of adaptation to disability and disease in health state valuation: A preliminary normative analysis. Social Science and Medicine, 55(12), 2149-2158. 
7. Brazier, J., Ratcliffe, J., Saloman, J., \& Tsuchiya, A. (2017). Measuring and valuing health benefits for economic evaluation ( $2 \mathrm{nd}$ ed.). New York: Oxford University Press.

8. Cieza, A., \& Stucki, G. (2005). Content comparison of healthrelated quality of life (HRQOL) instruments based on the international classification of functioning, disability and health (ICF). Quality of Life Research, 14(5), 1225-1237.

9. Dorman, P. J., Waddell, F., Slattery, J., Dennis, M., \& Sandercock, P. (1997). Are proxy assessments of health status after stroke with the EuroQol questionnaire feasible, accurate, and unbiased? Stroke, 28(10), 1883-1887.

10. Solli, O., Stavem, K., \& Kristiansen, I. S. (2010). Health-related quality of life in diabetes: The associations of complications with EQ-5D scores. Health and Quality of Life Outcomes, 8(1), 18.

11. Lin, F. J., Pickard, A. S., Krishnan, J. A., Joo, M. J., Au, D. H., Carson, S. S., et al. (2014). Measuring health-related quality of life in chronic obstructive pulmonary disease: Properties of the EQ-5D-5L and PROMIS-43 short form. BMC Medical Research Methodology, 14(1), 78.

12. Finch, A. P., Brazier, J. E., \& Mukuria, C. (2018). What is the evidence for the performance of generic preference-based measures? A systematic overview of reviews. The European Journal of Health Economics, 19(4), 557-570.

13. Kangwanrattanakul, K., \& Phimarn, W. (2019). A systematic review of the development and testing of additional dimensions for the EQ-5D descriptive system. Expert Review of Pharmacoeconomics \& Outcomes Research, 19(4), 431-443.

14. Gamst-Klaussen, T., Gudex, C., \& Olsen, J. A. (2018). Exploring the causal and effect nature of EQ-5D dimensions: An application of confirmatory tetrad analysis and confirmatory factor analysis. Health and Quality of Life Outcomes, 16(1), 153.

15. Nordlund, A., Ekberg, K., \& Kristenson, M. (2005). EQ-5D in a general population survey-A description of the most commonly reported EQ-5D health states using the SF-36. Quality of Life Research, 14(4), 1099-1109.

16. Johnson, J. A., \& Coons, S. J. (1998). Comparison of the EQ-5D and SF-12 in an adult US sample. Quality of Life Research, 7(2), $155-166$.

17. Richardson, J., Khan, M. A., Iezzi, A., \& Maxwell, A. (2012). Cross-national comparison of twelve quality of life instruments:
MIC Paper 1: Background, questions, instruments. Research paper 76. Retrieved 29 November, 2019, from https://aqol.com.au/paper s/researchpaper76.pdf

18. Hauke, J., \& Kossowski, T. (2011). Comparison of values of Pearson's and Spearman's correlation coefficients on the same sets of data. Quaestiones GEOGRAPHICAE, 30(2), 87.

19. O'brien, R. M. (2007). A caution regarding rules of thumb for variance inflation factors. Quality \& Quantity, 41(5), 673-690.

20. Lamu, A. N., \& Olsen, J. A. (2016). The relative importance of health, income and social relations for subjective well-being: An integrative analysis. Social Science \& Medicine, 152, 176-185.

21. Shapley, L. S. (1953). A value for n-person games. In H. W. Kuhn \& A. W. Tucker (Eds.), The theory of games (Vol. II, pp. 307317). Princeton: Princeton University Press.

22. Shorrocks, A. F. (2013). Decomposition procedures for distributional analysis: A unified framework based on the Shapley value. The Journal of Economic Inequality, 11(1), 99-126.

23. McFadden, D. (1974). The measurement of urban travel demand. Journal of Public Economics, 3(4), 303-328.

24. Smith, T. J., \& McKenna, C. M. (2013). A comparison of logistic regression pseudo R2 indices. Multiple Linear Regression Viewpoints, 39(2), 17-26.

25. Fox-Rushby, J. (2005). First steps to assessing semantic equivalence of the EuroQol instrument: Results of a questionnaire survey to members of the EuroQol Group. In P. Kind, R. Brooks, \& R. Rabin (Eds.), EQ-5D concepts and methods: A developmental history (pp. 35-52). Dordrecht: Springer.

26. Rand-Hendriksen, K., Augestad, L. A., Kristiansen, I. S., \& Stavem, K. (2012). Comparison of hypothetical and experienced EQ-5D valuations: Relative weights of the five dimensions. Quality of Life Research, 21(6), 1005-1012.

Publisher's Note Springer Nature remains neutral with regard to jurisdictional claims in published maps and institutional affiliations. 